\title{
New type of transverse moving box mechanism for pot seedling transplanting machine
}

\author{
Maile Zhou, Zhengyu Hua, Jingyuan Wang, Lei Wang, Yun Zhao, Daqing Yin* \\ (College of Engineering, Northeast Agricultural University, Harbin 150030, China)
}

\begin{abstract}
The existing moving box mechanism pot seedling transplanting machine on the market moves too fast when the picked seedling is collected, which negatively impacts the seedling picking performance. In order to improve the performance, two types of variable speed continuous moving box schemes were designed in this study. The first scheme was to apply a spiral-gear moving box spiral shaft with sine curve characteristics in the box moving mechanism, whereas the second one was to change the circular gear in the moving box into an elliptical gear with a speed shifting transmission mechanism. The working mechanism of the mechanical structure was analyzed, and the kinematic model was established. A dynamic analysis of the slider mechanism was performed. A virtual prototype was established according to agronomic parameters, and the virtual prototype experiments were conducted in ADAMS. The physical prototype and the high-speed photography experiment were performed on the test bench of a transplanting machine frame. The theoretical analysis, virtual prototype and physical prototype test results were consistent, which verified the validity of the theoretical model, virtual prototype and physical prototype and ensured the feasibility of the system.
\end{abstract}

Keywords: transplanting machine, rice transplanter, pot seedling, moving box mechanism, variable speed moving box, spiral shaft, elliptic gear

DOI: $10.25165 /$ j.ijabe.20181102.2867

Citation: Zhou M L, Hua Z Y, Wang J Y, Wang L, Zhao Y, Yin D Q. New type of transverse moving box mechanism for pot seedling transplanting machine. Int J Agric \& Biol Eng, 2018; 11(2): 70-75.

\section{Introduction}

Pot seedling transplanting has been widely popularized due to its largely increased yield per unit area. Research of the seedling transplanting machine has also been rapid. Seedling transplanting consists of three processes (picking, transporting and planting seedlings). The picking seedling step directly affects transporting and planting. Whether the box moving mechanism can achieve accurate and quantitative feeding of the seedlings is one of the key factors that affects the precision of seedling picking of the transplanting machine and is an important part of studies on transplanting equipment ${ }^{[1,2]}$.

Fully automatic seedling transplanting machines are primarily classified into the crank rocker type and rotary type, and the seedling picking approaches are divided into the seedling picking type and soil picking type ${ }^{[3-5]}$. Using the seedling transplanter PF100 model made by the Japan Iseki Corporation as an example, the core component in the matched transverse moving box mechanism is an ordinary spiral shaft. The machine conducts a uniform linear motion on a spiral shaft through a sliding sleeve and drives the seedling box to move transversely. Taking the

Received date: $2016-09-25$ Accepted date: 2017-05-17

Biographies: Maile Zhou, Lecturer, research interests: mechanization technology and equipment of transplanting. Email: zhoumaile@126.com; Zhengyu Hua, Master candidate, research interests: transplanting equipment. Email: 1264051248@qq.com; Jingyuan Wang, Master candidate, research interests: transplanting equipment. Email: 421247000@qq.com; Lei Wang, Master candidate, research interests: transplanting equipment. Email: 714523895@qq.com; Yun Zhao, Professor, research interests: transplanting equipment. Email: zhaoyun@neau.edu.cn.

*Corresponding author: Daqing Yin, PhD, Associate Professor, research interests: transplanting equipment. College of Engineering, Northeast Agricultural University, Harbin 150030, China. Tel: +86-451-55190971, Email: 63900962@qq.com. transplanting machine manufactured by the China Jilin Xinhuayu Agriculture Machinery Corporation as another example, the box moving mechanism comprises a transmission shaft, a spiral shaft and a transverse shaft for seedling feeding. Uniform transverse seedling feeding is transmitted by a slider on the spiral shaft to the transverse shaft of the seedling feeding; this process is stable and reliable. There are two types of transverse shifting mechanisms on the current market: the constant velocity type and the intermittent type. Rice transplanters adopt variable speed types of box moving mechanisms, and the seedling picking approach uses the needle tearing seedling block. Transverse movement of the seedling box will not affect the seedling quality during the seedling picking process. However, the seedlings are independent in each hole during transplanting. When the seedling picking frequency is too high, the constant velocity type transverse moving box mechanism is prone to leakage or damage of the seedling pot soil if the seedling box lateral displacement is larger than $1 / 6$ of the pot width at the seedling stage, causing unreliable seedlings and reducing the feeding success rate. On the other hand, the intermittent moving box will form a large inertia force during the seedling process and will produce strong vibrations and noise, which limits the quantity of seedlings picked per unit time and reduces the work efficiency ${ }^{[6]}$. Therefore, it is necessary to take a compromise approach based on the continuous moving box by lowering the speed of the box's movements during the seedling picking process to ensure that the moving distance is less than $1 / 6$ of the pot width. This approach will not make the seedling box inertia force too large and will ensure the reliability of seedling picking $^{[7-9]}$.

To meet the requirements of the above moving box, the following two schemes were put forward: first, changing the spiral shaft expansion line from a straight line to a sine curve based on the original linear oscillation, and second, changing the round gear in the transverse moving box mechanism into an elliptic gear to 
form a variable speed drive and achieve a transverse moving box in a low-speed movement at the seedling picking stage to ensure the reliability of seedling picking. The above two schemes were patented [the pot seedling transplanting variable velocity moving box mechanism (201310010615.8) and the double helix shaft of the variable speed transverse transfer box of a seedling transplanting machine $(201310010614.3)]^{[10,11]}$.

\section{Structural characteristics and working mechanism}

Figure 1 depicts the transverse moving box mechanism for the pot seedling transplanting machine. For the transverse moving box, the power is transmitted through bevel gear transmission 4 to driving wheel 2, which drives driven wheel 1 to rotate. Spiral shaft 3 and driven wheel 1 are fixedly connected and drive slider 9 and sliding sleeve 8 , which move along the helical shaft above spiral shaft 3 . Sliding sleeve 8 is fixedly connected to seedling box 10 to drive its transverse movement. The shaft of driving wheel 2 is connected with the shaft of the chain wheel through coupling 5, and the power is transported to transplanting mechanism 7 through chain transmission 6 , which is the power source of transplanting mechanism 7.

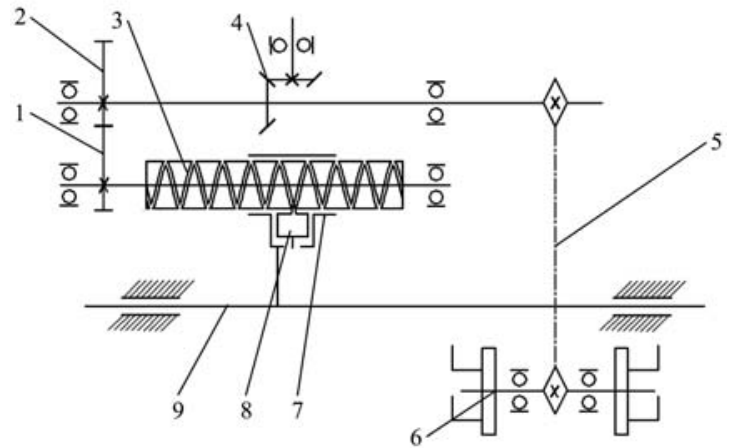

1. Driven wheel 2. Driving wheel 3. Spiral shaft 4. Bevel gear drive

5. Chain drive 6. Transplanting mechanism 7. Sliding sleeve 8 . Slider 9. Seedling box

Figure 1 Schematic of the transverse moving box mechanism

2.1 Working principle of the variable speed double helix shaft in the moving box mechanism

The comparison between the double helix shaft of the seedling transplanting machine transverse moving box and the ordinary double spiral shaft expansion line is shown in Figure 2. The moving box double helix shaft slide shaft expands with two spiral grooves of equal width so that their spirals are in opposite directions with the same pitch width in a continuous phase. The angle of the two spiral grooves differs by $180^{\circ}$. The ordinary double helix shaft expansion is linear, the slider moves along the spiral groove, and the seedling box transverse motion has a constant linear velocity. Conversely, the variable speed double helix shaft has an expansion based on the sinusoidal oscillation of the original line (linear), which guarantees that the slider speed is continuously changing and realizes a transverse moving box with continuous variable speeds. The design of the variable transverse double helix shaft achieves a sinusoidal motion of the slider around the original constant speed line with a speed variation as follows: low, medium, high speed, medium speed and back to low speed, alternating in circulation. The acceleration variation is also continuous, which guarantees stability of the movement. The low speed region falls within the seedling picking stage, which ensures that the seedling box transverse displacement is less than $1 / 6$ of the pot width.
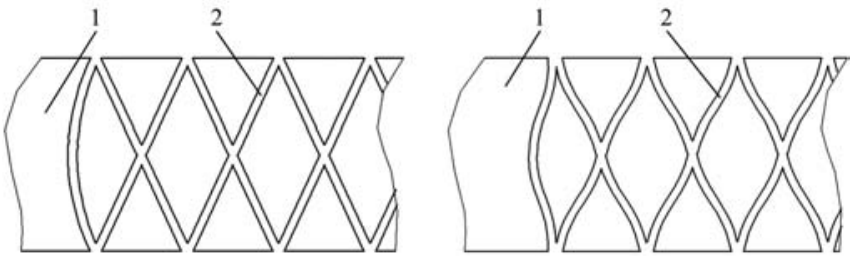

a. Ordinary double helix expansion b. Variable speed double helix expansion 1. Shaft body 2. Spiral groove

Figure 2 Ordinary and variable speed double helix expansion

\subsection{Working principle of elliptical gear transmission in the moving box mechanism}

This structure does not change the spiral shaft, and the expansion line of the spiral shaft is linear. The ordinary round gear transmission was replaced with an elliptical gear to drive the spiral shaft performance in a uniform circular motion of variable speed $^{[12,13]}$. Based on the variable speed rotation of the spiral shaft, the slider moves transversely with variable speed and drives the seedling box with variable speed displacement. When the seedling box is at low speed, the machine completes the seedling picking transplanting action. This structure can also ensure that the box in the process of transverse movement has a lower speed compared to the conventional moving box to ensure the quality of seedling picking.

\section{Kinematic analysis of the transverse moving box mechanism}

A kinematic analysis was performed on the slide of the spiral shaft to acquire the kinematic model of the box moving mechanism. Based on this kinematic model, the variable spiral shaft slide and elliptical gear drive variable speed mechanism was designed.

\subsection{Design of the variable speed shaft spiral slide}

The variable speed of the spiral shaft slide changes the slide block transverse displacement speed due to a slide curvature change. According to the operation requirements of the pot seedling transplanting machine, the movement speed and displacement time curve of the moving box mechanism designed by the author is shown in Figure 3. Its variable speed equation is

$$
v_{1}=\Delta v \cos (\omega t)+v
$$

where, $v$ is the original speed of the moving box and $\Delta v$ is the difference between the maximum displacement speed and the original speed. The high speed rotational transplanting mechanism picks seedlings twice while rotating once. Therefore, $\omega=\frac{4 \pi}{T}$ where $T$ is the time required for the transplanting mechanism to rotate once.

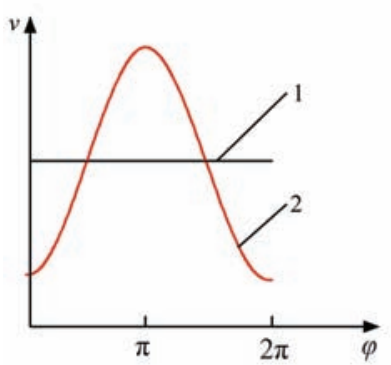

a. Velocity-time curve

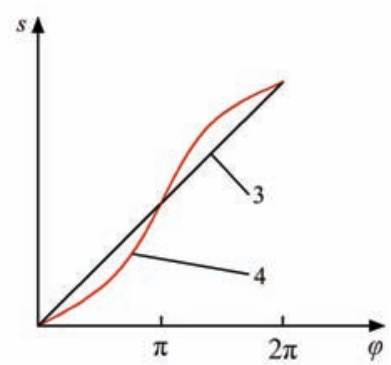

b. Displacement-time curve
1. Original shifting moving box velocity curve 2. Variable speed moving box velocity curve 3 . Original moving box displacement curve 4 . Variable speed shifting box displacement curve

Figure 3 Variable speed motion analysis 
Based on the variable speed equation, the displacement time equation is

$$
S(t)=\int_{0}^{t} v_{1} \mathrm{~d} t=\frac{\Delta v}{\omega} \sin (\omega t)+v t+c
$$

As discussed above, the displacement-time curve can be obtained from the rotational sinusoidal curve. The sinusoidal curve before the rotation is

$$
S^{\prime}(t)=\kappa \sin (\omega t)
$$

where, $\kappa$ is the speed variable coefficient. When $\kappa=1$, the seedling machine has a constant speed transverse movement.

Based on the rotation equation, the starting point $\left(x_{1}, y_{1}\right)$ is rotated to $\left(x_{2}, y_{2}\right)$, as follows:

$$
\left\{\begin{array}{l}
x_{2}=x_{1} \cos (\varphi)-y_{1} \sin (\varphi) \\
y_{2}=x_{1} \sin (\varphi)+y_{1} \cos (\varphi)
\end{array}\right.
$$

where, $\varphi$ is the rotational angle during the change in rotation and $\varphi=\pi / 2-\varphi_{s}$, where $\varphi_{s}$ is the spiral angle.

The curve equations after rotation are

$$
\left\{\begin{array}{l}
X=t \sin \left(\varphi_{s}\right)-\kappa \sin (\omega t) \cos \left(\varphi_{s}\right) \\
Y=t \cos \left(\varphi_{s}\right)+\kappa \sin (\omega t) \sin \left(\varphi_{s}\right)
\end{array}\right.
$$

\subsection{Elliptic gear transmission design}

The second scheme is to change the round helical gear that drives the shifting shaft into an elliptical gear box mechanism. The principle of this scheme is to use the elliptical gear driving the transmission ratio changing process, which drives the spiral shaft to rotate at a variable speed to achieve continuous variable speed motion of the seedling box. According to the actual demand, the minimum speed is half of the original moving speed, and the maximum speed is three times the minimum speed as follows: $k=1 /(2+\sqrt{3})=0.2679$. The elliptical gear modulus is 2.25 , and the tooth number is 23 .

The pitch curve equation of the elliptic gear transmission is

$$
r_{1}=\frac{A\left(1-k^{2}\right)}{1-k \cos \varphi_{1}}
$$

where, $\varphi_{1}$ is the active elliptical gear angle, the range is 0 to $2 \pi$ and $A$ is the major axis length of the elliptical gear.

The equation of the elliptic gear transmission ratio $i_{12}$ is

$$
i_{12}=\frac{1-2 k \cos \varphi_{1}+k^{2}}{1-k^{2}}
$$

When the driving elliptical gear rotation angle changes from $\varphi_{11}$ to $\varphi_{12}$, the angle of the driven elliptic gear $\varphi_{2}$ is

$$
\varphi_{2}=\int_{\varphi_{11}}^{\varphi_{12}} \frac{1}{i_{12}} \mathrm{~d} \varphi_{1}=\int_{\varphi_{11}}^{\varphi_{12}} \frac{1-k^{2}}{1+k^{2}-2 k \cos \varphi_{1}} \mathrm{~d} \varphi_{1}
$$

The transplanting machine test bench developed by our team was used as a carrier with a high-speed rotary rice pot seedling transplanting mechanism to test the seedling picking parts. The rice pot seedling transplanting mechanism is shown in Figure 4. The commonly used rice pot dish has 14 transverse points and 29 vertical points, and the center distance of the pot body is $20 \mathrm{~mm}^{[14]}$. Based on the test bench transmission ratio distribution, we can determine that in a single cycle, the input shaft rotates one cycle, the spiral shaft rotates one circle, the transplanting mechanism completes the transplanting process, and the seedling box transverse movement distance is equal to the center distance of the rice pot plate.

At the beginning of the seedling picking process of the transplanting mechanism, the rotation angle of the active driving elliptical gears is $\varphi_{11}$; at the end of the seedling picking process, the elliptic gear rotation angle is $\varphi_{12}$. From Equation (8), the seedling box transverse moving distance during picking seedling is

$$
\Delta h=\frac{20}{2 \pi} \int_{\varphi_{11}}^{\varphi_{12}} \frac{1-k^{2}}{1+k^{2}-2 k \cos \varphi_{1}} \mathrm{~d} \varphi_{1}
$$

The rotary rice pot seedling transplantation mechanism from the beginning to the end of the seedling process involves a rotation angle of the active elliptical gear of $15^{\circ}$. According to the driving relationship between the elliptical gear at the start and end of seedling picking, the position of the active elliptical gear is determined as a constant $\varphi_{12}-\varphi_{11}$. When $\frac{\left(\varphi_{12}+\varphi_{11}\right)}{2}=180^{\circ}$, the value of $\Delta h$ is the minimum and $\varphi_{11}=150^{\circ}$ and $\varphi_{12}=210^{\circ}$ were obtained. Using Equation (9), the seedling picking period was calculated and the transverse displacement of the seedling box as $\Delta h=2.1 \mathrm{~mm}$.

Based on the principle of the ordinary moving box mechanism, when the spiral shaft rotates, the slider is restricted by the slide track of the spiral shaft and the sliding sleeve; thus, it can only move transversely ${ }^{[15]}$ as shown in Figure 5.
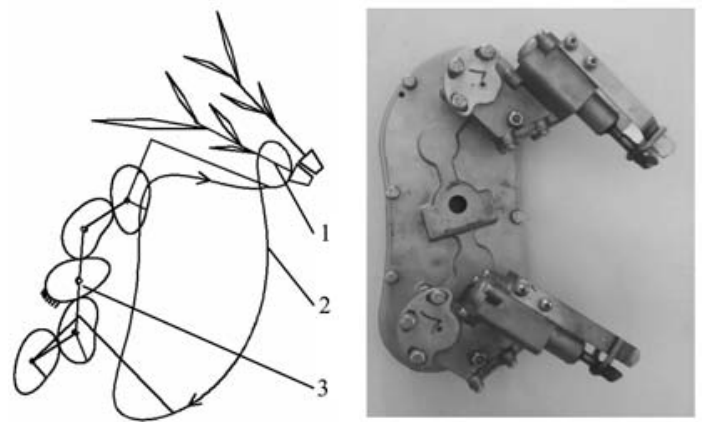

1. Pot seedling 2. Track 3. Transplanting mechanism

Figure 4 High speed rice pot seedling transplanting mechanism

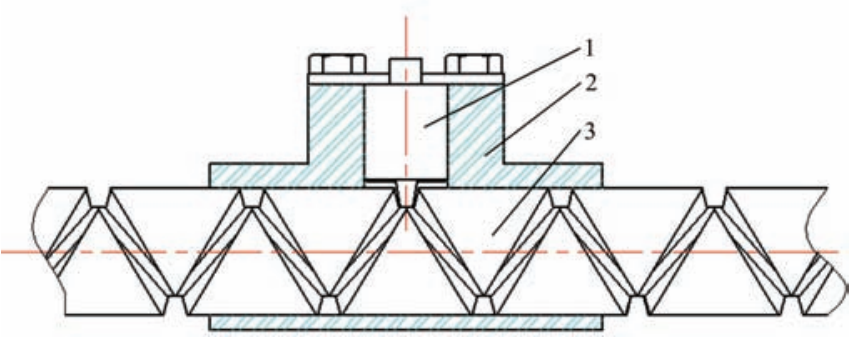

1. Slider 2. Sliding sleeve 3. Spiral shaft

Figure 5 Sliders and spiral shaft installation process

The speed and the acceleration of the variable speed moving box mechanism are continuously changing, which is different from the common moving box mechanism. Instantaneous acceleration direction at each moment occurs at the tangent direction of the contact surface. The force analysis of the slider is shown in Figure 6.

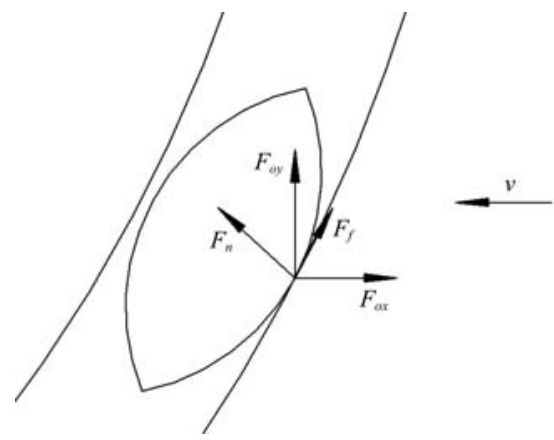

Figure 6 Force analysis of the slider 
The instantaneous force equation is

$$
\left\{\begin{array}{l}
\sum F_{x}=F_{o x}+F_{f} \cdot \sin \alpha-F_{n} \cdot \cos \alpha-m x=0 \\
\sum F_{y}=F_{o y}+F_{f} \cdot \cos \alpha+F_{n} \cdot \sin \alpha=0
\end{array}\right.
$$

where, $\alpha$ is the friction angle between the spiral groove wall and the YOZ plane. $F_{f}$ is the sliding fraction, $F_{n}$ is the positive pressure, and $F_{o x}$ and $F_{o y}$ are the forces of the seedling box on the slide force.

Because $F_{f}=F_{n} \tan \varphi$, (10) can be converted to

$$
\left\{\begin{array}{l}
F_{o x}+F_{n} \tan \varphi \sin \alpha-F_{n} \cos \alpha=m x \\
F_{o y}+F_{n} \tan \varphi \cdot \cos \alpha-F_{n} \cdot \sin \alpha=0
\end{array}\right.
$$

According to Equation (11), $F_{n}$ is obtained as follows:

$$
F_{n}=\left(F_{o x}-m x\right) \cdot \frac{\cos \varphi}{\cos (\alpha+\varphi)}
$$

The value of $F_{o x}$ is equal to the sliding friction in the sliding groove of the seedling box and is linearly correlated to the mass of the seedling box, which is

$$
F_{o x}=m g \tan \varphi_{1}
$$

$F_{n}$ and $F_{o y}$ can be obtained as follows:

$$
\begin{gathered}
F_{n}=m \cos \varphi \frac{g \tan \varphi_{1}-x}{\cos (\alpha+\varphi)} \\
F_{o y}=m\left(g \tan \varphi_{1}-x\right) \frac{\sin (\alpha-\varphi)}{\cos (\alpha+\varphi)}
\end{gathered}
$$

According to the characteristics of the isokinetic exercise, the seedling box transverse acceleration can be obtained as follows:

$$
\ddot{x}=\left[\kappa \sin \left(\omega t+\alpha_{0}\right)\right]=\kappa \omega \cos \left(\omega t+\alpha_{0}\right)
$$

Corresponding to the lowest point of the velocity in the seedling picking stage (i.e., the minimum extreme point) and corresponding to the first order of the continuous function when it equals $0, \omega t+\alpha_{0}=\frac{3}{2} \pi$. Finally, the following equations can be obtained:

$$
\begin{gathered}
F_{n}=m \cos \varphi \frac{g \tan \varphi_{1}-\kappa \omega \cos \left(\omega t+\alpha_{0}\right)}{\cos (\alpha+\varphi)} \\
F_{o y}=m\left[g \tan \varphi_{1}-\kappa \omega \cos \left(\omega t+\alpha_{0}\right)\right] \frac{\sin (\alpha-\varphi)}{\cos (\alpha+\varphi)}
\end{gathered}
$$

Using the high speed rice pot seedling transplanting mechanism as an example, the period between the contact of the clip with the seedling and the removal of the seedling corresponds to the lowest relative speed. The relationship between $F_{n}$ and $t$ is shown in Figure 7.

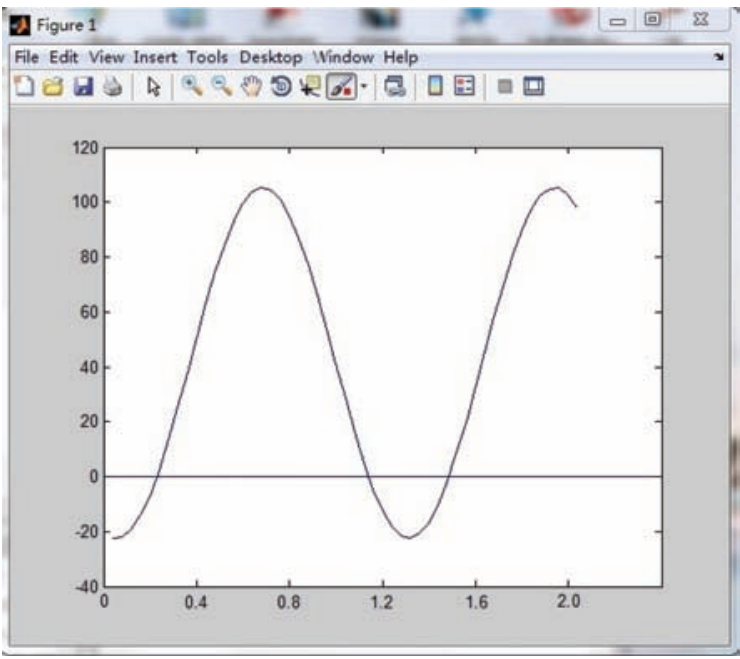

Figure 7 Relationship between $F_{n}$ and $t$

\section{Computer-aided analysis and virtual test}

Using three dimensional (3D) computer-aided design (CAD) software Unigraphics (UG) modeling, the parts were assembled through the constraints ${ }^{[16,17]}$. As shown in Figure 8 , the virtual prototype of the two new variable speed moving box mechanisms is displaced.

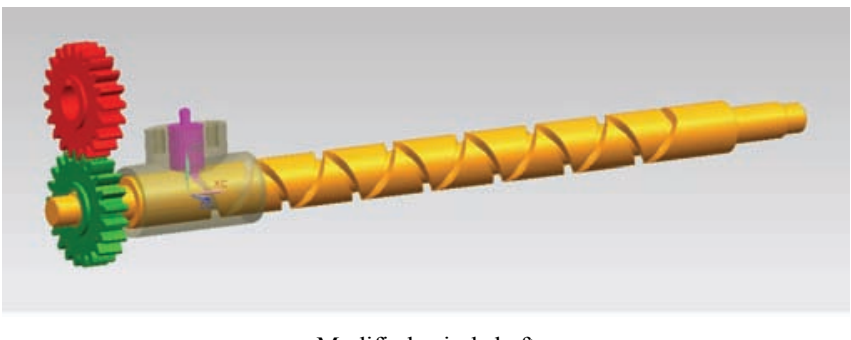

Modified spiral shaft

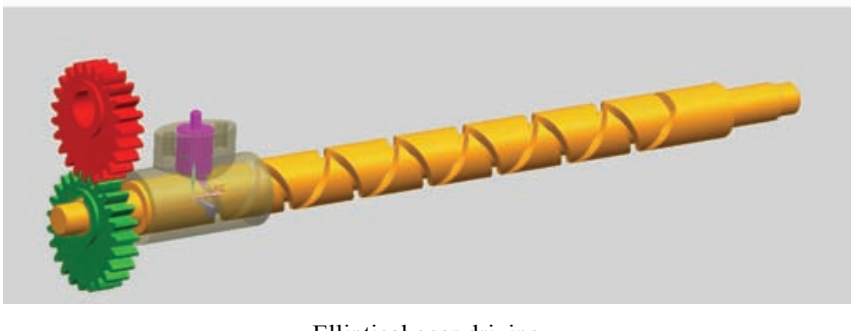

Elliptical gear driving

Figure 8 Virtual prototype of a new gear moving box mechanism

By introducing the 3D model of UG into automatic dynamic analysis of mechanical systems (ADAMS), a virtual prototype test was performed on the moving box mechanism after adding constraints and providing driving. The slider displacement and velocity curve can be measured in the virtual prototype test, as shown in Figure 9.

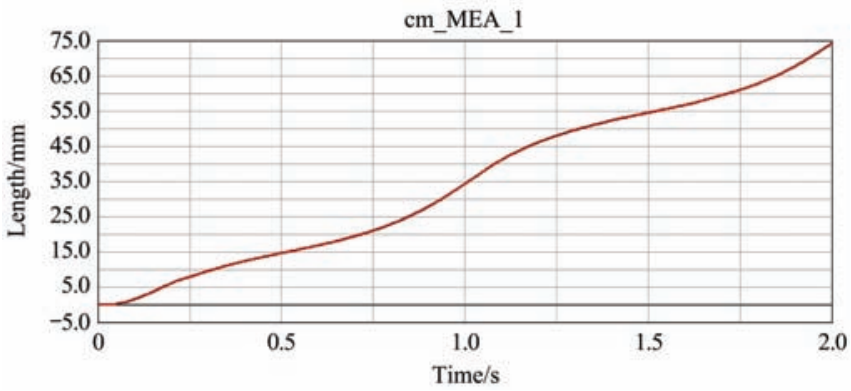

a. Virtual prototype test displacement curve

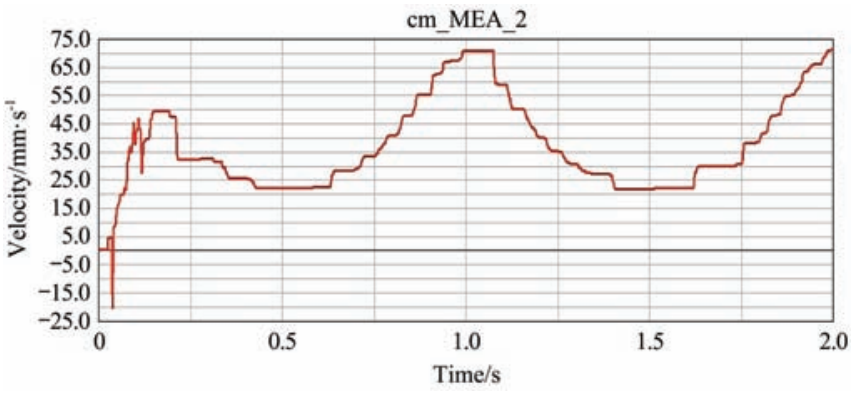

b. Virtual prototype test velocity curve

Figure 9 Virtual prototype test displacement and velocity curve

The theoretical optimal trajectory coincides with the trajectory of the virtual prototype test. Additionally, the measured slider velocity curve and the theoretical velocity curve were consistent, which verified the correctness of the theoretical analysis and ensured the feasibility of the mechanism ${ }^{[18,19]}$. 


\section{Experiment}

\subsection{Bench test}

To verify the feasibility of the new type of transverse seedling feeding mechanism design, the distance of transverse movement during seedling picking and the transverse displacement and velocity of the moving box were measured. The variable velocity transverse moving box mechanism was installed on the transplanting machinery test bench. The test table input speed was $200 \mathrm{r} / \mathrm{min}$, and the slider was marked. The transverse moving box mechanism motion was shot with Phantom high speed photography equipment, and the analysis was conducted on high speed videos with the Phantom Camera Control software to obtain high speed photography of the transverse moving box mechanism motion model. The novel transverse moving box mechanism prototype is shown in Figure 10.

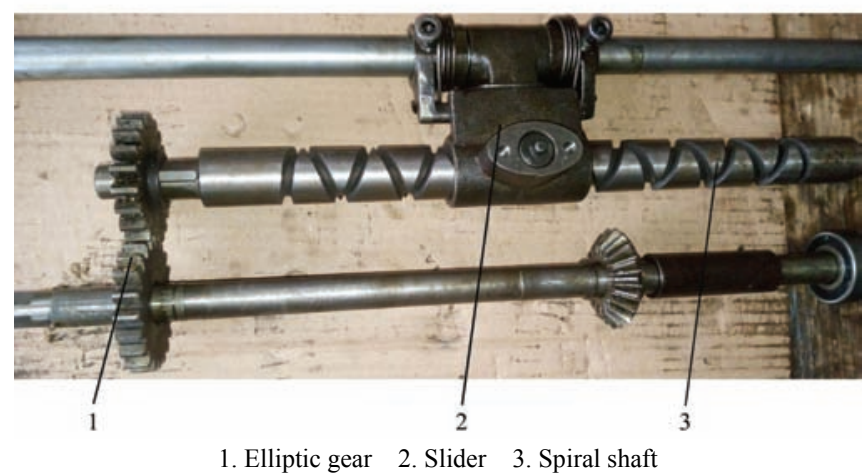

Figure 10 Prototype of a new type of transverse moving box mechanism

According to the high-speed photography of the physical prototype of the new type of transverse moving box mechanism, the measurement and analysis of velocity and marker displacement were performed using the Phantom Camera Control software. The displacement curve and velocity curve models of the transverse moving box mechanism were obtained as shown in Figure 11. It was observed that the transverse moving box mechanism displacement and velocity curve of the physical prototype test and the theoretical analysis results were consistent, which verified the feasibility of the application of the transverse moving box mechanism.

\subsection{Prototype test}

A new type of transverse moving box mechanism was proposed, which was equipped with the ordinary type rice pot transplanting machine. The seedling picking experiment was conducted by high-speed photography as shown in Figure 12.

The high-speed photography video was analyzed as shown in Figure 13. Using rice pot seedling transplanting as an example, the pot width was $18 \mathrm{~mm}$. Within the seedling picking period, the transverse movement of the box moving mechanism in the picking seedling stage was at the bottom of the speed curve, and the seedling box transverse moving distance was $2.05 \mathrm{~mm}$. Compared with the traditional seedling box mechanism with a transverse moving distance of $3.33 \mathrm{~mm}$, the new mechanism reduced the displacement by $1.28 \mathrm{~mm}$, which was far below $1 / 6$ of the pot width. The seedling age of rice seedlings was $35 \mathrm{~d}$, at a transplanting mechanism rotation speed of $150 \mathrm{r} / \mathrm{min}$ (there were 300 seedling picking actions per minute), the average success rate of seedling picking was $99.2 \%$, ensured the reliability of seedling picking.

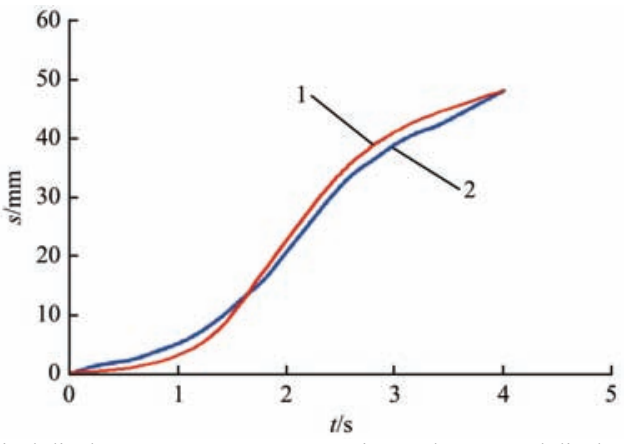

1. Theoretical displacement curve $\quad 2$. Experimental measured displacement curve

a. Seedling box displacement curve

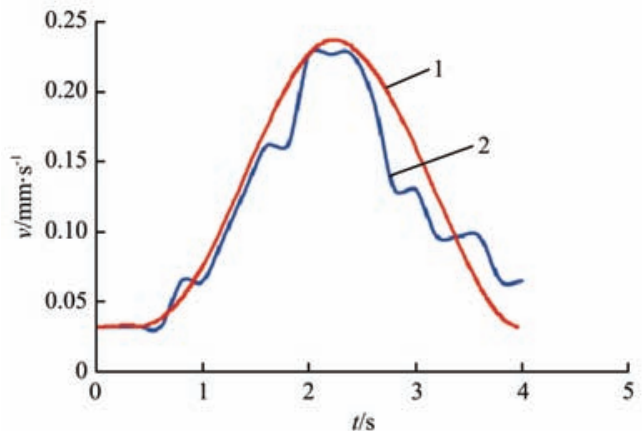

1. Theoretical velocity curve 2 . Experimental velocity displacement curve

$$
\text { b. Seedling box velocity curve }
$$

Figure 11 Displacement and velocity curve of the moving box mechanism with variable speeds

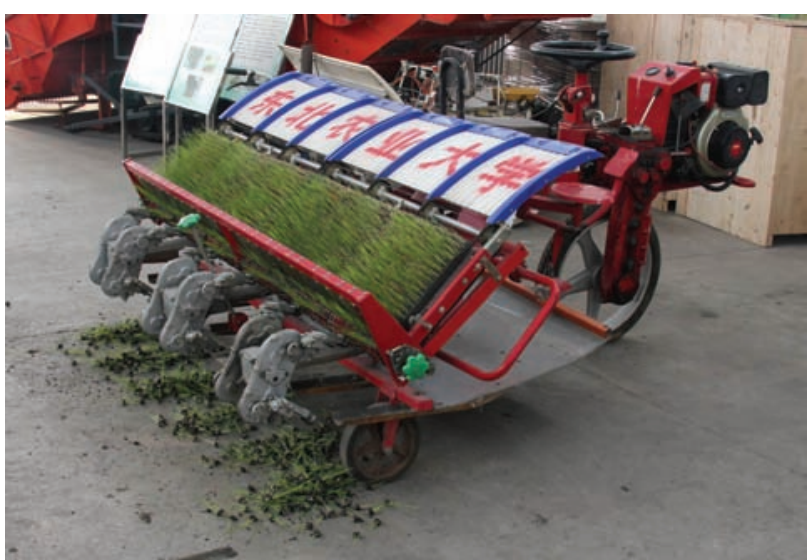

Figure 12 Ordinary type rice pot seedling transplanting machine

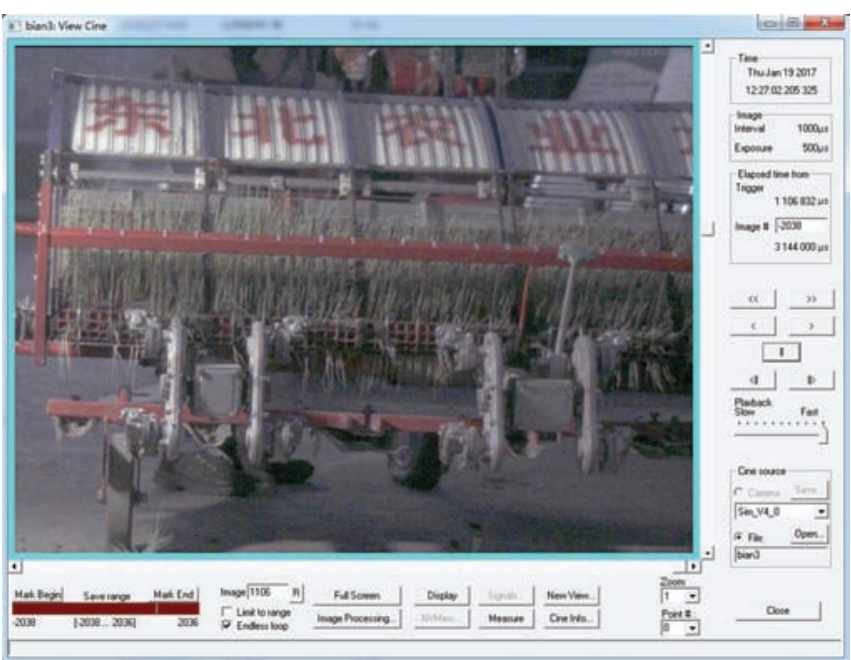

Figure 13 High-speed photography video analysis 


\section{Conclusions}

1) The rotary transplanting mechanism needs to reduce speed without stopping of the box movement during seedling picking. One way to address this requirement is to change the moving box shaft spiral line of the transplanting machine box mechanism with characteristics of a sine curve and put forward a new type of spiral shaft, such as a variable speed spiral shaft; another method is to replace the circular gear with an elliptic gear in the box moving mechanism. Thus, a new type of gear box moving mechanism was proposed to achieve a continuous non-constant velocity moving box, which created the foundation for high speed seedling transplanting.

2) The working mechanism of the moving box mechanism was analyzed, and a mechanical analysis of the slider was performed. By reducing the seedling box impact load, the variable speed moving box mechanism could greatly improve the seedling picking success rate.

3) The 3D modeling of the mechanism was completed. The movement characteristics of the mechanism were basically in agreement with the theoretical analysis results, which met the requirement of high speed pot seedling transplanting.

4) A high speed photography test was performed on the physical prototype of the new transverse moving box mechanism. The displacement and velocity curves of the seedling box transverse motion were obtained. The results from the theoretical analysis, the test results of the virtual prototype and the high-speed photography were basically consistent, which verified their correctness.

5) The seedling picking experiment was completed combined with ordinary type rice pot transplanting machine, the average success rate of seedling picking was $99.2 \%$, ensured the reliability of seedling picking.

\section{Acknowledgements}

This research was supported by the National Key Research and Development Program of the 13th Five-year Plan (Grant No. 2017YFD070802-2), the China Special Fund for Agro-Scientific Research in the Public Interest (Grant No. 201203059-01), the National Key Technology R\&D Program (Grant No. 2014BAD06B-1-05), the Open Fund Projects of Zhejiang Province Planting Equipment Technology Key Laboratory (Grant No. 2013E10013-06), the National Natural Science Foundation of China (Grant No. 51775104), and by the National Key Technology R\&D Program (Grant No. 2014BAD06B01-13).

\section{[References]}

[1] Yang W Z, Zhao Y, Li G, Yu G H. Optimization design of a high speed rice transplanter box rotary spiral shaft orbit shift machine. Transactions of the CSAM, 2003; 34(6): 167-168.

[2] Thomas E V. Development of a mechanism for transplanting rice seedlings. Mechanism and Machine Theory, 2002; 37(4): 395-410.

[3] Zhao Y, Zhou M L, Zhao X, Sun J S. Rotary type rice pot seedling transplanting mechanism: China Patent, No. ZL201310317551.6, 2015.12.16.

[4] Zhou M L, Sun L, Du X, Xin L. Optimal design and experiment of rice pot seedling transplanting mechanism with planetary bezier gears. Transactions of the ASABE, 2014; 57(6): 1537-1548.

[5] Yu X X, Zhao Y, Chen B C, Zhou M L, Zhang H, Zhang Z C. Development status and prospect of transplanting machinery. Transactions of the CSAM, 2014; 45(8): 44-53.

[6] Yang W Z, Yang Y D, Zhang Y, Zhao Y. Four axis high-speed rice transplanter moving box mechanism design principle. Chinese Agricultural Mechanization, 2005; 5: 67-69.

[7] Nagasaka Y, Umeda N, Kanetai Y, Taniwaki K, Sasaki Y. Autonomous guidance for rice transplanting using global positioning and gyroscopes. Computers and Electronics in Agriculture, 2014; 43(3): 223-234.

[8] Kumar G V P, Raheman H. Development of a walk-behind type hand tractor powered vegetable transplanter for paper pot seedlings. Biosystems Engineering, 2011; 110(2): 189-197.

[9] Kumar G V P, Raheman H. Automatic feeding mechanism of a vegetable transplanter. Int J Agric \& Biol Eng, 2012; 5(2): 20-27.

[10] Zhao Y, Xin L, Na M J, Liu X L. Seedling transplanting non constant velocity continuous moving box mechanism. China Patent, No. 201310010615.8, 2013-04-10.

[11] Zhao Y, Xin L, Wang L J. Variable speed transverse shifting box double helix shaft of pot seedling transplanting machine. China Patent, No. 201310010614.3, 2013-04-10

[12] Bair B-W. Computer aided design of elliptical gears. Journal of Mechanical Design, 2002; 24(4): 787-793.

[13] Bair B-W. Computer aided design of elliptical gears with circular-arc teeth. Mechanism and Machine Theory, 2004; 39(2): 153-168.

[14] Zhou M L. Mechanism analysis and optimization design of the transplanting mechanism of high speed rice seedling transplanting mechanism with Bessel gear. Zhejiang: Zhejiang Sci-Tech University, 2014.

[15] Li B F. Agricultural Mechanics. Beijing: China Agriculture Press, 2003.

[16] Zhao Y. Outline of modern design methods of agricultural machinery. Journal of Northeast Agricultural University, 2012; 43(2): 1-5.

[17] Jalón J G D, Serna M A, Avilés R. Computer method for kinematic analysis of lower-pair mechanisms. Mechanism and Machine Theory, 1981; 16(5): 94-114

[18] van de Werken J. The development of the finger-tray automatic transplanting system. Journal of Agricultural Engineering Research, 1991; 50: $51-60$

[19] Zhao Y. Analysis and synthesis of agricultural machinery. Beijing: Mechanical Industry Press, 2008. 